\title{
Nutrition and cognitive function in the elderly
}

\author{
Gerd Faxén Irving
}

Department of Geriatric Medicine, Huddinge University Hospital, Stockholm, Sweden

\begin{abstract}
There are complex associations between nutrition and dementia disorders. Dementia, especially Alzheimer's disease (AD), is associated with an increased risk of protein-energy malnutrition. Weight loss and eating problems increase with the severity and progression of dementia, e.g. in AD. Whether malnutrition per se has a negative impact on cognitive function or whether nutritional intervention may improve cognitive function in demented people is still not proven. The few studies so far performed show that nutritional therapy leads to improved nutritional status. Data on the effects on cognitive function after nutritional intervention in demented populations are sparse and inconclusive. It cannot be excluded that effects on cognitive function may also be accomplished. Data from epidemiological studies suggest that some nutrients, e.g. fatty acids, may interfere with the development of dementia.
\end{abstract}

Keywords: cognition, dementia, elderly, liquid supplements, nutrition.

\section{Introduction}

The mean life expectancy and proportion of elderly people in the population will continue to increase during the coming decades. As a consequence, the number of age-related diseases, e.g. dementia disorders, is likely to increase. Today, around 7\% ( 150000 ) of elderly Swedish subjects over the age of 65 years are assumed to suffer from dementia. People with dementia, especially Alzheimer's disease (AD), have been identified as being at particularly high risk of malnutrition (1). Cross-sectional studies show that demented elderly weigh less and have lower body mass index (BMI) than cognitively intact elderly people (2). Around $12-50 \%$ of institutionalized demented patients suffer from malnutrition $(3,4)$. The frequency is reported to be higher in AD than in vascular dementia. Weight loss seems to be a predictor of mortality in subjects with $\mathrm{AD}$, whereas weight gain appears to have a protective effect (5). It is unclear whether proteinenergy malnutrition (PEM) precedes or follows dementia (6). Studies suggest that weight loss frequently occurs in the first stages of the disease. It is uncertain whether the weight loss in demented patients is due to inadequate dietary intake or dementia-related metabolic disturbances (3, 6-9). There are several possible reasons for inadequate food intake, such as apraxia (no longer knowing how to eat), agnosia (no longer recognizing food as food), forgetting to eat, changes in olfaction and taste, difficulty with shopping and meal preparation, depression and refusal to eat (10). It has been suggested that some AD patients have hyperactivity-related energy losses, but studies of energy metabolism in connection with dementia, performed with different techniques, have produced conflicting results $(11-14)$.

The effects of dietary intervention in elderly people with dementia have not been sufficiently elucidated. There are relationships between cognition and nutrition (Fig. 1), but the mechanisms are unclear. To find ways to improve the quality of life for the elderly it is important to understand the relationships between nutrition and cognition.

\section{Nutritional intervention studies}

Is it possible to improve nutritional status in demented individuals by nutritional intervention, and is it possible to affect cognitive function in this way? To the author's knowledge four studies have evaluated the effects of nutritional formula supplementation on nutritional status in study groups with only demented subjects. Of these, only two studies have evaluated possible effects on cognition. These studies are briefly reviewed. 


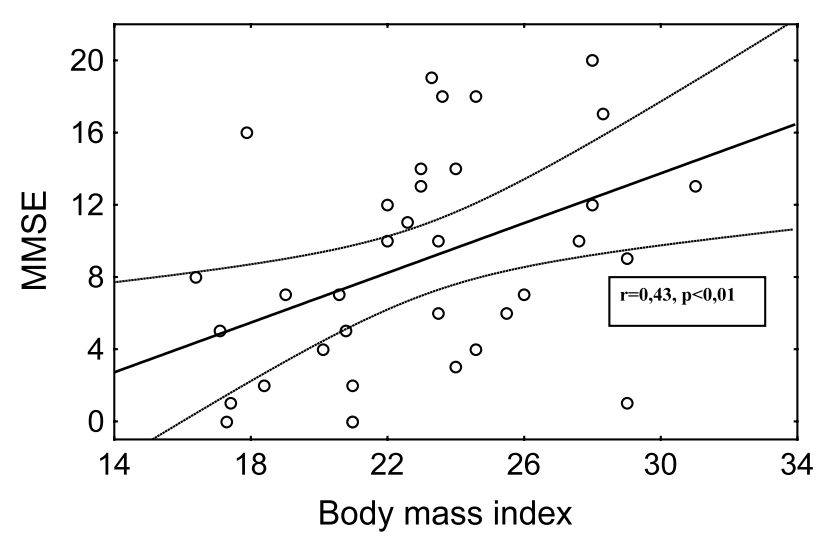

Fig. 1. Correlation between body mass index and Mini Mental State Examination (MMSE) in 34 elderly residents in two units of group living for the demented (see ref. 18).

\section{Malnourished demented patients in a British psychiatric hospital}

In a randomized controlled trial, Carver and Dobson (15) investigated dietary therapy for dementia patients with PEM. Of nearly 300 patients, 80 $(27 \%)$ were underweight. Of these, 46 were included in a randomized treatment study using liquid dietary supplements of $600 \mathrm{kcal} \mathrm{day}^{-1}$ for 12 weeks. The intervention group gained an average of $3.5 \mathrm{~kg}$ in weight, while the control group maintained a stable weight. No evaluation of functional capacity, cognitive function or mortality was reported.

\section{Dutch psychogeriatric nursing home residents}

Wouters-Wesseling et al. (16) recently reported a double-blind, placebo-controlled, 12 week intervention study on 42 psychogeriatric nursing-home patients, $\geq 60$ years of age, with BMI $<23$ for men or $<25$ for women. A liquid nutrition supplement, $270 \mathrm{kcal} \mathrm{day}^{-1}$, resulted in a significant improvement in body weight $(2.2 \mathrm{~kg})$, homocysteine, vitamin $B_{1}$, thiamine diphosphate (TDF), vitamin $B_{6}$, vitamin $B_{12}$, folate and vitamin $D$ in the intervention group compared with the placebo group. No significant difference was observed in activities of daily living (ADL). The effect on cognitive function was not reported.

Intervention with additional vitamins and minerals. Abalan et al. (17) studied the effects of supplements on cognition in a randomized controlled pilot study of 29 patients with dementia disorders. Fifteen individuals received vitamins, minerals and liquid supplements for 105 days. From day 36 to 105 the dose of the liquid supplement was doubled.
This resulted in an energy increase from 400 to 800 kcal day ${ }^{-1}$. Fourteen subjects served as controls. At the end of the study the treatment group was reported to have a significant improvement in Mini Mental State Examination (MMSE) score $(+3.5$ from a mean MMSE of 11.5 at the start) compared with the control group $(-2.7$ from a mean of 9.7 at start). Effects on weight or other nutritional parameters were not reported in this study.

\section{Demented elderly subjects residing in group living}

To study the effects of nutritional intervention on body weight, cognition and ADL, a controlled, non-randomized study was performed on elderly demented people housed in group living for the demented (18). Twenty-two residents in one unit received oral liquid supplements $\left(\right.$ Semper $\left.^{\circledR}\right), 410$ kcal day ${ }^{-1}$ for 5 months, while 14 residents in another unit served as controls. Weight increased by $3.4 \mathrm{~kg}$ in the intervention group, while the weight remained unchanged in the control group (Fig. 2). The rate of cognitive decline was not affected. This finding of weight gain is in line with the previously mentioned reports.

Regarding this study on nutritional intervention in group living for the demented, it cannot be excluded that more sensitive instruments may have registered the small signs of functional and cognitive improvement that were noted by relatives and staff. One of the many issues that need to be addressed is whether early nutritional intervention in subjects with milder forms of dementia would have other effects. The few studies performed so

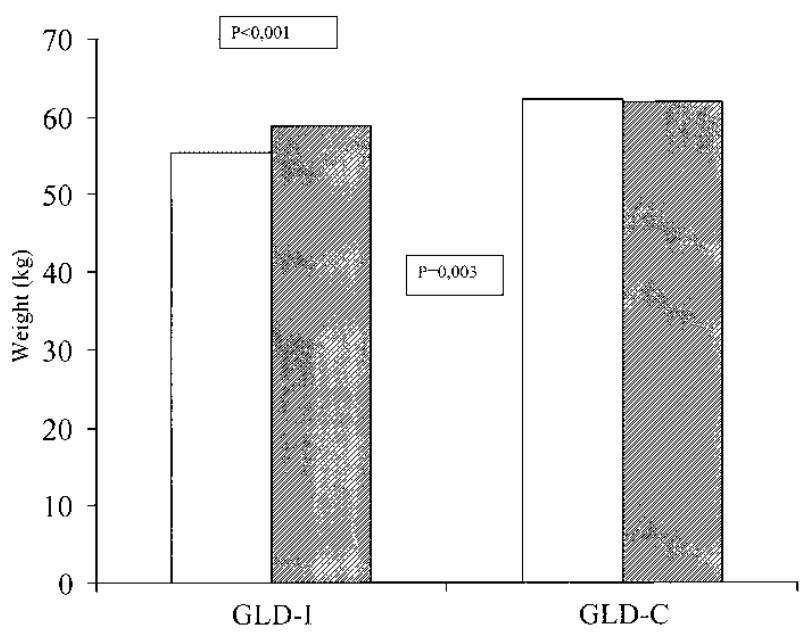

Fig. 2. Change in weight after 6 months of intervention with oral supplements $\left(400 \mathrm{ml} 410 \mathrm{kcal} \mathrm{day}^{-1}\right)$ in residents in group living for the demented (GLD). I: intervention group $(n=22)$; C: control group ( $n=14)$; open bars: 0 months; hatched bars: 6 months. 
far show that nutritional therapy leads to improved nutritional status. It cannot be excluded that effects on cognitive functions may also be accomplished.

\section{Prevention of dementia by nutrients}

\section{Omega-3 fatty acids}

The role of dietary fat in dementia has gained increasing interest. Fatty acids could be involved in cognition through several mechanisms. In addition to providing vascular protection, the omega-3 (n-3) fatty acids could reduce inflammation in the brain and may play a specific role in brain development and the regeneration of nerve cells. Results from the Rotterdam study, a single-centre, prospective, population-based study (19), showed that high intakes of total fat, saturated fat and cholesterol were associated with an increased risk of dementia, whereas an inverse association between fish consumption and $\mathrm{AD}$ was found. Recently, BarbergerGateau et al. (20) published a cohort study with 7 years of follow-up. They concluded that elderly people who eat fish or seafood at least once a week have a lower risk of developing dementia, including AD.

\section{Antioxidants}

Oxidative processes have been suggested to contribute to the development of AD. Therefore, antioxidants that counteract the detrimental effects of oxidative stress may reduce the risk.

It is unknown whether dietary intake of vitamin E and other antioxidant nutrients prevents the development of $\mathrm{AD}$. Prospective and longitudinal studies show different results. The strongest evidence for an association with cognition has been reported for vitamins C and E. In June 2002 Engelhart et al. (21) presented new results from the Rotterdam study (see above), in which high intakes of vitamins $\mathrm{C}$ and $\mathrm{E}$ were associated with a lower risk of $\mathrm{AD}$. The number of studies that argue for a protective effect of antioxidants on cognitive performance is increasing, but more prospective studies are needed.

The preventive effect of alcohol has also been discussed and epidemiological data from the Rotterdam group led to the conclusion that a moderate intake prevents dementia. Today there is growing evidence for associations between hypertension, hyperlipidaemia and the incidence of dementia later in life, suggesting that healthy eating may have protective effects.

\section{Conclusion}

There are associations between nutrition and dementia disorders but they are complex. A few intervention studies with liquid supplements in demented elderly people resulted in improved nutritional status. More studies are needed to evaluate not only the effects on nutritional parameters, but also the effects on cognitive performance, ADL functions and quality of life. Other interesting questions for the future include whether early nutritional intervention affects cognition, and whether specific nutrients, e.g. n-3 fatty acids, prevent the development of dementia.

\section{References}

1. Gillette-Guyonnet S, Nourhashémi F, Andrieu S, et al. Weight loss in Alzheimer disease. Am J Clin Nutr 2000; 71(Suppl): S637-42.

2. Faxén-Irving G, Andrén-Olsson B, Cederholm T. Nutritional and cognitive status in elderly subjects living in service flats and the effect of nutrition education on personnel. Gerontology 1999; 45: 187-94.

3. Sandman PO, Adolfsson R, Nygren C, et al. Nutritional status and dietary intake in institutionalized patients with Alzheimer's disease and multiinfarct dementia. J Am Geriatr Soc 1987; 35: 31-8.

4. Burns A, Marsh A, Bender DA. Dietary intake and clinical, anthropometric and biochemical indices of malnutrition in elderly demented patients and nondemented subjects. Psychol Med 1989; 19: 383-91.

5. White H, Pieper C, Schmader K. The association of weight change in Alzheimer's disease with severity of disease and mortality: a longitudinal analysis. J Am Geriatr Soc 1998; 46: 1223-7.

6. Barrett-Connor E, Edelstein SL, Corey-Bloom J, Wiederhot WC. Weight loss precedes dementia in community-dwelling older adults. J Am Geriatr Soc 1996; 44: 1147-52.

7. Wang SY, Fukagawa N, Hossain M, Ooi WL. Longitudinal weight changes, length of survival and energy requirements of long term care residents with dementia. Am Geriatr Soc 1997; 45: 1189-95.

8. Grundman M, Corey-Bloom J, Jernigan T, et al. Low body weight in Alzheimer's disease is associated with mesial temporal cortex atrophy. Neurology 1996; 46: 1585-9.

9. Singh S, Mulley GP, Losowsky MS. Why are Alzheimer patients thin? Age Ageing 1988; 17: 21-8.

10. Morris CH, Hope RA, Fairburn CG. Eating habits in dementia. Br J Psychol 1989; 154: 801-6.

11. Poehlman ET, Roman VD. Energy expenditure, energy intake, and weight loss in Alzheimer disease. Am J Clin Nutr 2000; 71(Suppl): S650-5.

12. Poehlman ET, Toth MJ, Goran MI, et al. Daily energy expenditure in free-living non-institutionalized Alzheimer's patients: a doubly labeled water study. Neurology 1997; 48: 997-1002. 
13. Donaldson KE, Carpenter WH, Toth MJ, et al. No evidence for a higher resting metabolic rate in noninstitutionalized Alzheimer's disease patients. J Am Geriatr Soc 1996; 44: 1232-4.

14. Wolf-Klein GP, Silverstone FA, Lansey SC, et al. Energy requirements in Alzheimer's disease patients. Nutrition 1995; 11: 264-8.

15. Carver AD, Dobson AM. Effects of dietary supplementation of elderly demented hospital residents. J Hum Nutr Diet 1995; 8: 389-94.

16. Wouters-Wesseling W, Wouters AEJ, Kleijer CN, et al. Study of the effect of a liquid nutrition supplement on the nutritional status of psycho-geriatric nursing home patients. Eur J Clin Nutr 2002; 56: 245-51.

17. Abalan F, Manciet G, Dartigues J-F, et al. Nutrition and SDAT. Biol Psychiatry 1992; 31: 103-5.

18. Faxén-Irving G, Andrén-Olsson B, Geijerstam A, et al. The effect of nutritional intervention in elderly subjects residing in group-living for the demented. Eur $\mathbf{J}$ Clin Nutr 2002; 56: 221-7.
19. Kalmijn S, Launer LJ, Ott A, et al. Dietary fat intake and the risk of incident dementia in the Rotterdam study. Ann Neurol 1997; 42: 776-82.

20. Barberger-Gateau P, Letenneur L, Deschamps V, et al. Fish, meat, and risk of dementia: cohort study. Br Med J 2002; 325: 932-3.

21. Engelhart MJ, Geerlings MI, Ruitenberg A, et al. Dietary intake of antioxidants and risk of Alzheimer disease. JAMA 2002; 287: 3223-9.

\section{Gerd Faxén Irving}

Department of Geriatric Medicine

Huddinge University Hospital

SE-I4I 86 Stockholm

Sweden

Tel: + $4685858607 \mid$

Fax: + 46858586075

E-mail: gerd.faxen-irving@hs.se 